'L Favre, 'KY Saudan, ${ }^{2 B}$ Corthésy, ${ }^{1} \mathrm{~J}$ Benyacoub. 'Nutrition \& Health, Nestlé Research Center; '2Immunology \& Allergy, State University Hospital, Lausanne, Switzerland

Background and Aims Transmission of immune competence from mothers to newborns is crucial for optimal development of neonate immune system. Maternal perinatal probiotics supplementation having been observed to be able to modulate this process, the goal of the present study was to investigate the importance of the time window of probiotics intervention (pregnancy/lactation) on early-life immune maturation and response to immunization.

Methods Pregnant C57/BL6 mice were supplemented with Bifido-

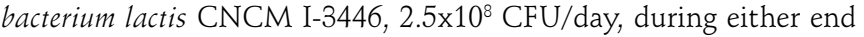
of gestation and lactation, end of gestation only or lactation only. Maltodextrin was given during both periods (placebo) or in replacement of probiotics when not administered. Immune maturation was assessed by measuring natural mucosal IgA production (ELISPOTs) at weaning and 6 weeks later. Pups were mucosally immunized at weaning, and again four weeks later, with live attenuated Salmonella typhimurium $\Delta$ aroA. Two weeks after the second immunization, specific antibody responses in serum were analyzed.

Results All probiotic regimens significantly enhanced natural IgA production in pups in comparison to placebo, an effect observable up to the end of study, 6 weeks post-weaning. Supplementation during end of pregnancy and lactation, or lactation only provided significantly highest values. Specific antibody titers tended to be potentiated by all three regimens in pups responding to immunization, with highest values being obtained after supplementation during both periods.

Conclusions This study further supports the benefit of maternal perinatal intervention with probiotics on neonatal immune maturation, moreover emphasizing that supplementation during both pregnancy and lactation is needed to achieve overall optimal effects.

\section{SEROTYPE AND ANTIMICROBIAL SUSCEPTIBILITY DISTRIBUTION OF INVASIVE STREPTOCOCCUS PNEUMONIAE ISOLATED FROM CHILDREN IN TURKEY}

doi:10.1136/archdischild-2012-302724.0268

${ }^{1} \mathrm{M}$ Ceyhan, ${ }^{2} \mathrm{~N}$ Gürler, ${ }^{3} Y$ Camcıoğlu, ${ }^{4} \mathrm{~A}$ Yaman, ${ }^{5} \mathrm{C}$ Özakın, Pneumo-Surveillance Study Group, M Gültekin, C Öztürk, Ş Aydemir, A Kiremitçi, F Aydın, Y Zer, H Uslu, L Şanal, D Perçin, H Güdücüoğlu. 'Department of Pediatric Infectious Diseases, Hacettepe University Faculty of Medicine, Ankara; ${ }^{2}$ Department of Microbiology and Clinical Microbiology, Istanbul University Istanbul Medical Faculty; ${ }^{3}$ Department of Pediatrics and Pediatric Diseases, Istanbul University Cerrahpasa Medical Faculty, Istanbul; ${ }^{4}$ Central Laboratory, Çukurova University Medical Faculty, Balcalı Hospital, Adana; ${ }^{5}$ Department of Clinical Microbiology, Uludağ University Medical Faculty, Bursa, Turkey

Streptococcus pneumoniae is a major cause of invasive infections. The aim of this study was to evaluate the serotype and antimicrobial susceptibility of invasive pneumococci isolated at 14 different centers in Turkey between January 2011-April 2012. Totally 79 clinical isolates from invasive infections were investigated, which were isolated from cerebrospinal fluid (CSF) (33, 42\%), blood (31, 39\%) and the other sterile body fluids $(15,19 \%)$. Susceptibility to penicillin, cefotaxime and erythromycin was determined by E-test (bioMerieux, France) according to CLSI standards. Latex agglutination method was used for determination of serogroups. Serotypes were determined by the capsular swelling (Quellung reaction) method (Denmark, Statens Serum Institute). It was found that most common serotypes among 79 strains were $19 \mathrm{~F}(12,15 \%), 6 \mathrm{~A}(7,9 \%), 23 \mathrm{~F}(5,6 \%), 6 \mathrm{~B}(4,5 \%)$, $19 \mathrm{~A}(4,5 \%)$ and $3(4,5 \%)$. For all invasive pneumococcal diseases, during the first 2 years of age, the potential coverage rates of PCV7, PCV10, and PCV13 were 47.8\%, 56.5\%, and $82.6 \%$, respectively; meanwhile $40.5 \%, 44.3 \%$, and $63.3 \%$ for the pediatrics age group (0-18). Serotypes 19F, 6A, 19A, 23F, 6B, 14 and 3 were predominate. All pneumococcal conjugate vaccine formulations cover these serotypes with the exception of serotype 19A which is covered only by PCV13. Serotype 19A has steadily increased in prevalence and become increasingly resistant to common antibiotic classes. Rational antibiotic use and vaccination of infants with pneumococcal conjugate vaccines should be considered as essential strategies for prevention of pediatric invasive infections in Turkey.

\section{SHIFTING SEROPOSITIVITY FOR HEPATITIS A IN CHILDREN IN ISTANBUL, TURKEY FROM 1996 TO 2011}

doi:10.1136/archdischild-2012-302724.0269

${ }^{1} \mathrm{RG}$ Sezer, ${ }^{1} \mathrm{~A}$ Bozaykut, ${ }^{1} \mathrm{~V}$ Akcan, ${ }^{1} \mathrm{C}$ Paketci, ${ }^{1} \mathrm{LP}$ Seren, ${ }^{2} \mathrm{G}$ Aydemir, ${ }^{2} \mathrm{IA}$ Tanju. ${ }^{1}$ Department of Pediatrics, Zeynep Kamil Maternity and Childrens' Disease Training and Research State Hospital; '2Department of Pediatrics, GATA Teaching Hospital, Istanbul, Turkey

Background Hepatitis A virus (HAV) is transmitted by the fecaloral route, and the epidemiology of HAV is associated with hygiene and socioeconomic status. However, due to improvements in living conditions, there is an epidemiological shift in HAV infection.

Methods In this study, we investigated the seropositivity for HAV in children aged between 2 and 18 years. In addition, we compared the results with previously reported seropositivity data from the same center in Uskudar, Istanbul, Turkey, from 1996.

Results The mean age of the 400 children was $7.9 \pm 3.7$ years (range: $2-18)$. Of the 400 serum samples collected, all were tested for antiHAV IgG, and 50 (12.5\%) were positive. The rates of anti-HAV seropositivity within the age groups of $2-<6,6-<10$ and $10-18$ years were determined. The seropositivity increased with increasing age: $11.5 \%$ in the 2 - to $<6$-year-old group and $13.2 \%$ in the 10 - to 18 -yearold group.

Conclusions There was a significant decline in the overall seropositivity for anti-HAV between 1996 and 2011 ( $p<0.001$ ), and the pediatric age group has a high risk of HAV infection.

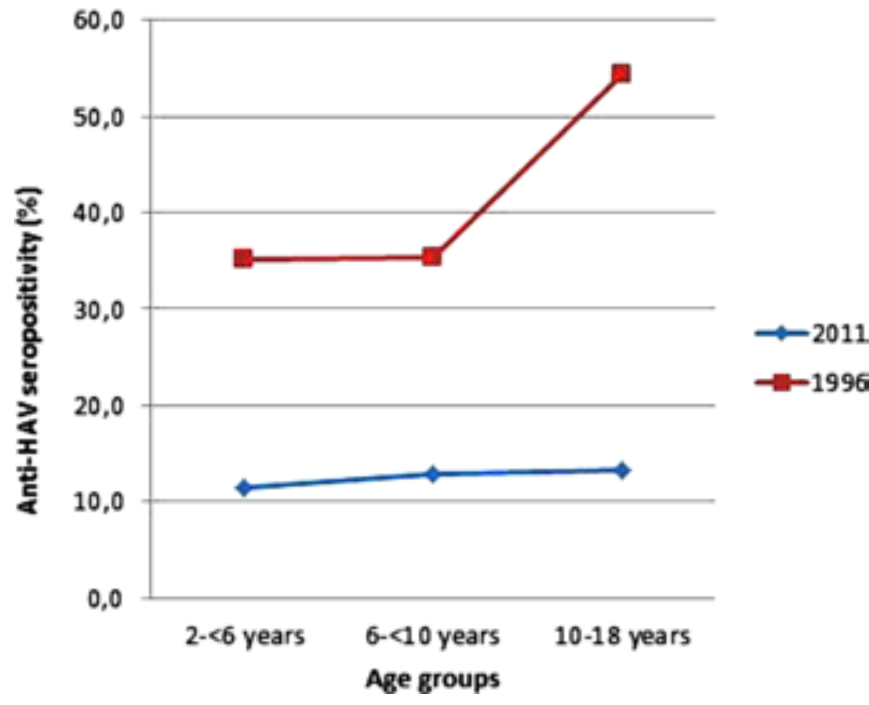

Abstract 269 Figure 1 Shifting seropositivity for Hepatitis A

In 1996, the overall seropositivity was $41.3 \%$. In the 1996 study, the seropositivity was $35.2 \%$ in 2 - to < 6 -year-old age group, $35.3 \%$ in the 6 - to $<10$-year-old age group and $54.3 \%$ in children older than 10 years. Given the serological shift over time, greater susceptibility and a persistent risk of exposure to HAV suggest that outbreaks are possible.

\section{H1N1 PANDEMIC: COMPARISON OF THE CLINIC PRESENTATION BETWEEN CANADA AND FRANCE IN CRITICALLY ILL CHILDREN}

doi:10.1136/archdischild-2012-302724.0270 